\title{
Boosting efficiency in light-driven water splitting by dynamic irradiation through synchronizing reaction and transport processes
}

Maximilian Sender, ${ }^{[a] \ddagger}$ Fabian L. Huber, ${ }^{[b] \ddagger}$ Maximilian C. G. Moersch, ${ }^{[a, b]}$ Daniel Kowalczyk, ${ }^{[a]}$ Julian Hniopek, ${ }^{[c, d]}$ Sarah Klingler, ${ }^{[e]}$ Michael Schmitt, ${ }^{[d]}$ Simon Kaufhold, ${ }^{[b]}$ Kevin Siewerth, ${ }^{[b]}$ Jürgen Popp, ${ }^{[c, d]}$ Boris Mizaikoff, ${ }^{[e]}$ Dirk Ziegenbalg*[a] and Sven Rau*[b]

[a] M. Sender, M. C. G. Moersch, Daniel Kowalczyk, Prof. Dr. D. Ziegenbalg Institute of Chemical Engineering Ulm University

Albert-Einstein-Allee 11, 89081 Ulm, Germany

E-mail: dirk.ziegenbalg@uni-ulm.de

[b] F. L. Huber, Kevin Siewerth, Dr. Simon Kaufhold, Prof. Dr. S. Rau Institute of Inorganic Chemistry I

Ulm University

Albert-Einstein-Allee 11, 89081 Ulm, Germany

E-mail: sven.rau@uni-ulm.de

[c] J. Hniopek, Prof. Dr. J. Popp

Department Spectroscopy \& Imaging

Leibniz Institute of Photonic Technology

Albert-Einstein-Str. 9, 07745 Jena, Germany

E-mail: juergen.popp@leibniz-ipht.de

[d] J. Hniopek, Prof. Dr. M. Schmitt, Prof. Dr. J. Popp

Institute of Physical Chemistry \& Abbe Center of Photonics

Friedrich Schiller University Jena

Helmholtzweg 4, 07743 Jena, Germany

E-mail: juergen.popp@uni-jena.de

[e] S. Klingler, Prof. Dr. B. Mizaikoff

Institute of Analytical and Bioanalytical Chemistry

Ulm University

Albert-Einstein-Allee 11, 89081 Ulm, Germany

E-mail: boris.mizaikoff@uni-ulm.de

¥ These authors contributed equally to this work

\section{Abstract}

This work elaborates the effect of dynamic irradiation on light-driven molecular water oxidation to counteract catalyst deactivation. It highlights the importance of overall reaction engineering to overcome limiting factors in artificial photosynthesis reactions. Systematic investigation of a homogenous three component ruthenium-based water oxidation system revealed significant potential to enhance the overall catalytic efficiency by synchronizing the timescales of photoreaction and mass transport in a capillary flow reactor. The overall activity could be improved by a factor of more than 10 with respect to the turnover number and a factor of 31 referring to the external energy efficiency by controlling the local availability of photons. Detailed insights into the mechanism of light driven water oxidation could be obtained using complementary methods of investigation like Raman, IR and UVvis/emission spectroscopy, unraveling the importance of avoiding high concentrations of excited photosensitizers. 


\section{Main Text}

Photocatalytic reactions have received tremendous attention from both academia and industry as sunlight presents an abundant and sustainable energy source. ${ }^{[1,2]}$ The light-driven splitting of water into oxygen and hydrogen - artificial photosynthesis - is a promising approach to supply renewable fuels and feedstock to the economy. ${ }^{[3]}$ Many heterogeneous and homogenous systems for the photochemical splitting of water have been developed and chemically optimized in regard to molecular components, buffer conditions and pH-ranges. ${ }^{[4-8]}$ Artificial photosynthetic reactions are so far usually broken down into two half reactions, which are studied separately: Oxidation of water to form oxygen, protons and electrons, and reduction of protons to yield hydrogen. ${ }^{[9]}$ The overall performance of photocatalytic reactions depends on a complex interplay between molecular properties of the involved chemical species, the environment around the active components and the presence of light of suitable intensity and wavelength. ${ }^{[10]}$ The highly ordered and synchronized mechanisms of biological photosynthesis can serve as a source of inspiration: to unleash the full potential of artificial photosynthetic catalysis, a thorough understanding of the interaction between the catalytic species, the micro- and macroscopic mass transport as well as the radiation field is necessary. ${ }^{[11]}$ Thus, catalyst development and reaction engineering need to be combined synergistically. ${ }^{[12-14]}$ Detailed investigations by Bonnet et al. into the mechanism of light driven water oxidation catalysis (WOC) with a molecular photosensitizer (PSm), $\left[\mathrm{Ru}(\mathrm{bpy})_{3}\right]\left(\mathrm{PF}_{6}\right)_{2}$, three different molecular catalysts ([Ru(bda)(isoq) $\left.)_{2}\right]$ (bda = 2,2'-bipyridine-6,6'-dicarboxylic acid, isoq = isoquinoline), $\mathrm{Co}\left(\mathrm{NO}_{3}\right)_{2}$ and [Ir $\left.\left(\mathrm{Cp}^{*}\right)(\mathrm{dmiz})(\mathrm{OH})_{2}\right]\left(\mathrm{Cp}^{*}=\right.$ pentamethylcyclopentadienyl, dmiz =1,3-dimethylimidazol-2-ylidene) $)$ and $\mathrm{Na}_{2} \mathrm{~S}_{2} \mathrm{O}_{8}(10 \mathrm{mM})$ as sacrificial electron-acceptor highlighted the complex interplay of various factors determining the overall catalytic activity. The effect of the ratio of the individual components and the light intensity were investigated in detail. It was found that the photocatalytic activity can be improved for the combination of $\left[\mathrm{Ru}(\mathrm{bpy})_{3}\right]\left(\mathrm{PF}_{6}\right)_{2}$ and $\left[\mathrm{Ru}(\mathrm{bda})(\mathrm{isoq})_{2}\right]$ by increasing concentration of both components. However, degradation under photocatalytic conditions of both molecular components via decomposition of the ligand scaffolds was identified as a major deactivation pathway. The light intensity was identified as a key parameter determining the rate of deactivation. High light intensities lead to an accumulation of $\mathbf{P S m}^{+}$which subsequently opens the degradation pathway. For a high activity, matching the kinetic rates of the excitation of the PSm with the subsequent electron transfer and/or the rate of oxygen formation is required. Given that the electron transfer is rate limiting, which causes the described accumulation of $\mathbf{P S m}^{+}$, tuning (decreasing) the rate of PSm excitation by lowering the incident photon flux represents a reaction engineering approach to control the lifetime of the system. As shown by Bonnet et al., this results in higher stability of the WOC systems. Thus, beside increasing the chemical stability of the involved molecular components, reaction engineering factors like the spatiotemporal availability of photons have to be taken into account in addition to chemical factors.

To understand the impact of changing irradiation conditions on the photocatalytic water oxidation, we adopted the well-known system consisting of $\left[R u(d p p)(p i c)_{2}\right]\left(\mathrm{PF}_{6}\right)_{2}(\mathbf{R u}(\mathbf{d p p}), \mathrm{dpp}=2,9$-di(pyridine-2'yl)-1,10-phenanthroline, $\mathrm{pic}=4$-picoline), which acts as single-site water oxidation catalyst, the photosensitizer $\left[\mathrm{Ru}(\mathrm{dceb})_{2}(\mathrm{bpy})\right]\left(\mathrm{PF}_{6}\right)_{2} \quad(\mathrm{PS}, \quad \mathrm{dceb}=$ diethyl[2,2'-bipyridine $]-4,4^{\prime}$-dicarboxylate, bpy $=2,2^{\prime}$-bipyridine) and $\mathrm{Na}_{2} \mathrm{~S}_{2} \mathrm{O}_{8}$ as sacrificial electron-acceptor in a solvent mixture of $96 \mathrm{v}-\%$ of aqueous $\mathrm{H}_{3} \mathrm{BO}_{3} / \mathrm{NaHCO}_{3}$ buffer (pH 6.5) and $4 \mathrm{v}-\%$ MeCN (Fig. 1 a). ${ }^{[15-17]}$ 

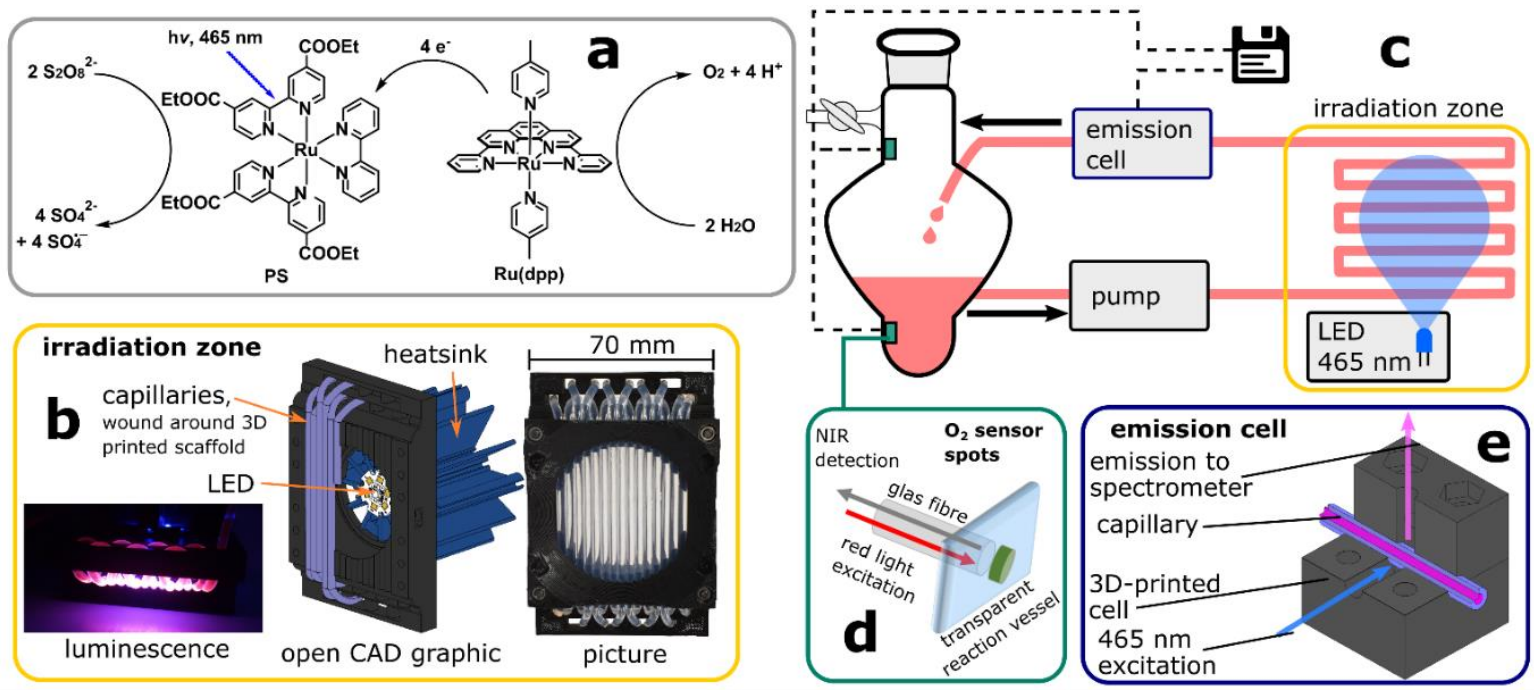

Figure 1. Illustration of the homogenous three component water oxidation system (a) and schematic depiction of the capillary flow-through-reactor setup ( $b-e)$.

To study the impact a of temporally changing availability of photons, a novel capillary flow-through reactor, operated in recycling mode, is used for WOC (Fig. 1). ${ }^{[18-22]}$ In this reactor system the reaction mixture is cycled from the storage vessel through a capillary which is wound in a 3D-printed scaffold. ${ }^{[23,24]}$ The parallel arrangement of the capillary creates a defined projection area that is adapted to the emission characteristics of the light source (Fig. $1 \mathbf{c}$ and b). Oxygen formation was monitored in solution as well as in the gas phase by an optical sensor (Fig. $1 \mathrm{~d}$ ). ${ }^{[18]}$ Integrity of the PS was monitored by tracing its emission in situ with a clamp-on emission cell (Fig. 1 e). Further details on the setup are given in the ESI.

The influence of the operating conditions on the catalytic performance was studied systematically by varying the flow rates of the reaction mixture and irradiation intensity of the light source. While passing through the capillary, the reaction solution passes through irradiated and non-irradiated parts of the reactor (compare Figure $1 \mathrm{~b}$ ). Varying the flow rate from 6 to $50 \mathrm{~mL} \mathrm{~min}^{-1}$ leads to average irradiation periods of 0.77 to $0.09 \mathrm{~s}$ and dark periods of 1.47 to $0.18 \mathrm{~s}$ (see Table 1; full details in ESI Table S1) and thus a lower average incident photon flux. Higher flow rates lead to shorter irradiation/dark periods and could thus be helpful to synchronize different reaction steps. Additionally, the light intensity could be adjusted through variation of the radiant power of the LED. Both parameters directly influence the reaction rate and with this also the interaction of mass transport and reaction rate.

The catalytic performance of the system was determined by four parameters: turnover number (TON, amount of product per catalyst), turnover frequency (TOF, TON per time interval), as well as external energetic $\left(\xi_{\mathrm{E}}\right)$ and photonic efficiencies $\left(\xi_{\mathrm{p}},\right)$, referring to the amount of oxygen generated per energy consumed or photons emitted by the light source (detailed description in ESI).

Table 1. Mean irradiation periods in the scaffold for different flow rates.

\begin{tabular}{lll}
\hline flow rates $/(\mathrm{mL} / \mathrm{min})$ & mean irradiation period $/ \mathrm{s}$ & Mean irradiation pauses $/ \mathrm{s}$ \\
\hline 6 & 0.77 & 1.47 \\
17 & 0.27 & 0.52 \\
21 & 0.22 & 0.42 \\
29 & 0.16 & 0.30 \\
50 & 0.090 & 0.18 \\
\hline
\end{tabular}


For other catalytic systems, an increase of irradiation intensity leads to an increase in catalytic performance, ${ }^{[25]}$ however, similar to the results of Bonnet et al., the results show a generally higher maximum TONs at lower irradiation intensities. This effect is most pronounced when high flow rates are applied (c.f. Fig. 2a). By changing the operating conditions, the TONs could be increased by a factor of more than 11 from $33\left(6 \mathrm{~mL} \mathrm{~min}^{-1}, 0.37 \mathrm{~W}\right)$ to $378\left(50 \mathrm{~mL} \mathrm{~min}^{-1}, 0.12 \mathrm{~W}\right)$. These changes in performance become more obvious when analyzing the efficiency parameters $\xi_{\mathrm{E}}$ and $\xi_{\mathrm{p}}$. A 25 -fold increase in external energetic efficiency from around $2 \mu \mathrm{mol} \mathrm{Wh} \mathrm{W}^{-1}$ to $49 \mu \mathrm{mol} \mathrm{Wh} \mathrm{h}^{-1}$ was found. $\xi_{\mathrm{p}}$ increases from $1 \%$ to $31 \%$, representing a 31-fold increase of the external photonic efficiency. The even more pronounced increase is attributed to the higher efficiency of the LED at lower radiant power. Both performance indicators give evidence that the impact of deactivation processes is reduced under such conditions.
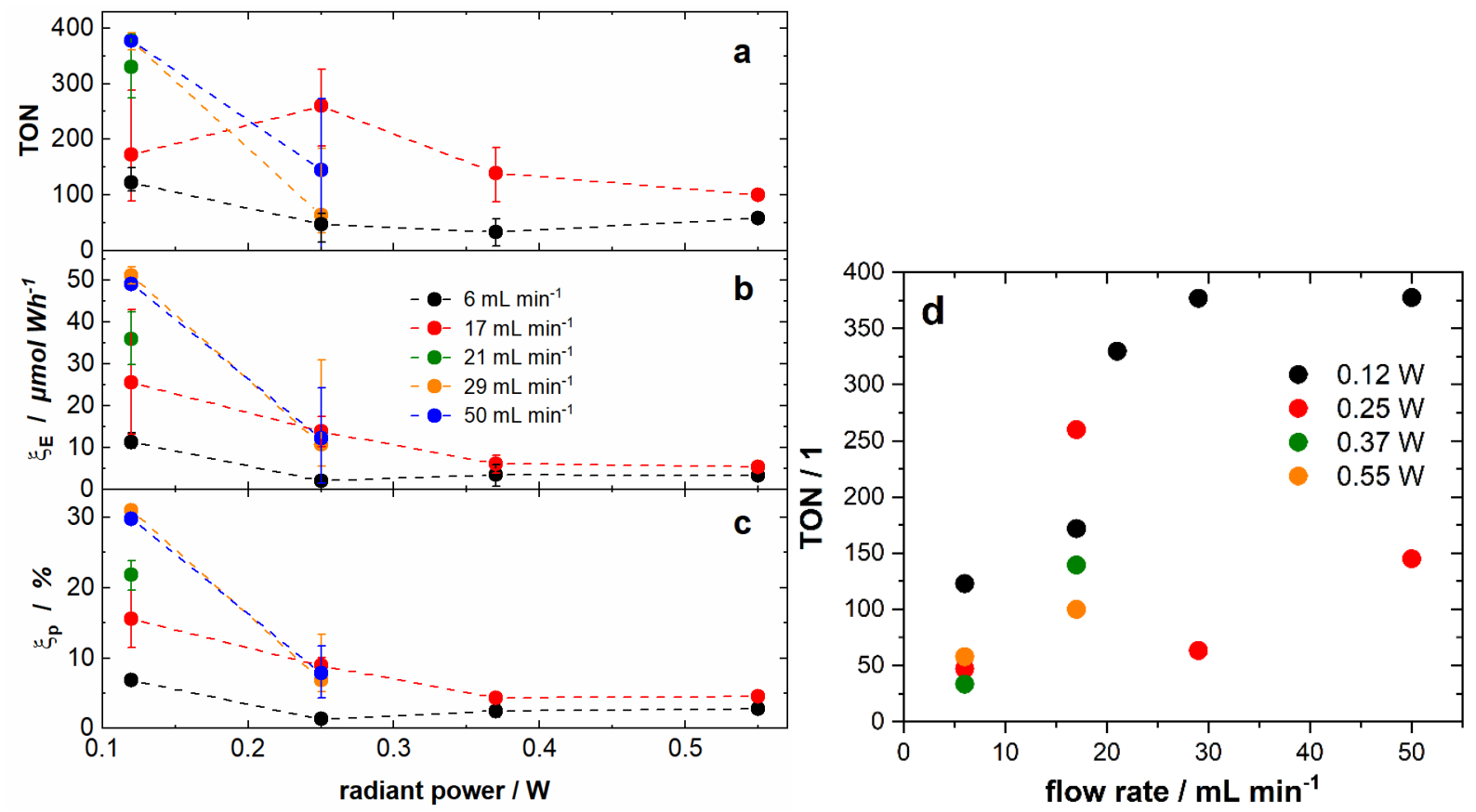

Figure 2: Impact of different flow rates and light intensities on the photocatalytic performance. Dotted lines added for visual clarity. $\boldsymbol{a}$ : Results given in maximum TON achieved, each point was measured at least three times. $\boldsymbol{b}$ : Results in external energetic efficiency $\left(\xi_{E}\right)$. c: Results in external photonic efficiency $\left(\xi_{p}\right)$. d: Maximum TON in dependence of the flow rate.

Time resolved oxygen measurements at different light intensities show a fast breakdown of the catalytic activity for high irradiation intensity (Figure 3). At a flow rate of $6 \mathrm{~mL} \mathrm{~min}$-1 $^{-1}$ and a radiant power of $0.55 \mathrm{~W}$ the maximum TON of 60 was reached in around $10 \mathrm{~min}$, while at $0.12 \mathrm{~W}$ radiant power the maximum TON of approximately 110 was reached after $65 \mathrm{~min}$ (Fig. 4 a). Accordingly, TOF at $0.55 \mathrm{~W}$ intensity reached $0.38 \mathrm{~s}^{-1}$ and declined fast after reaching maximum activity. While at $0.12 \mathrm{~W}$ a maximum TOF of only $0.09 \mathrm{~s}^{-1}$ was reached but maintained over a prolonged period (Fig. $4 \mathrm{~b}$ ). Measurements at different flow rates generally showed the same trend. 

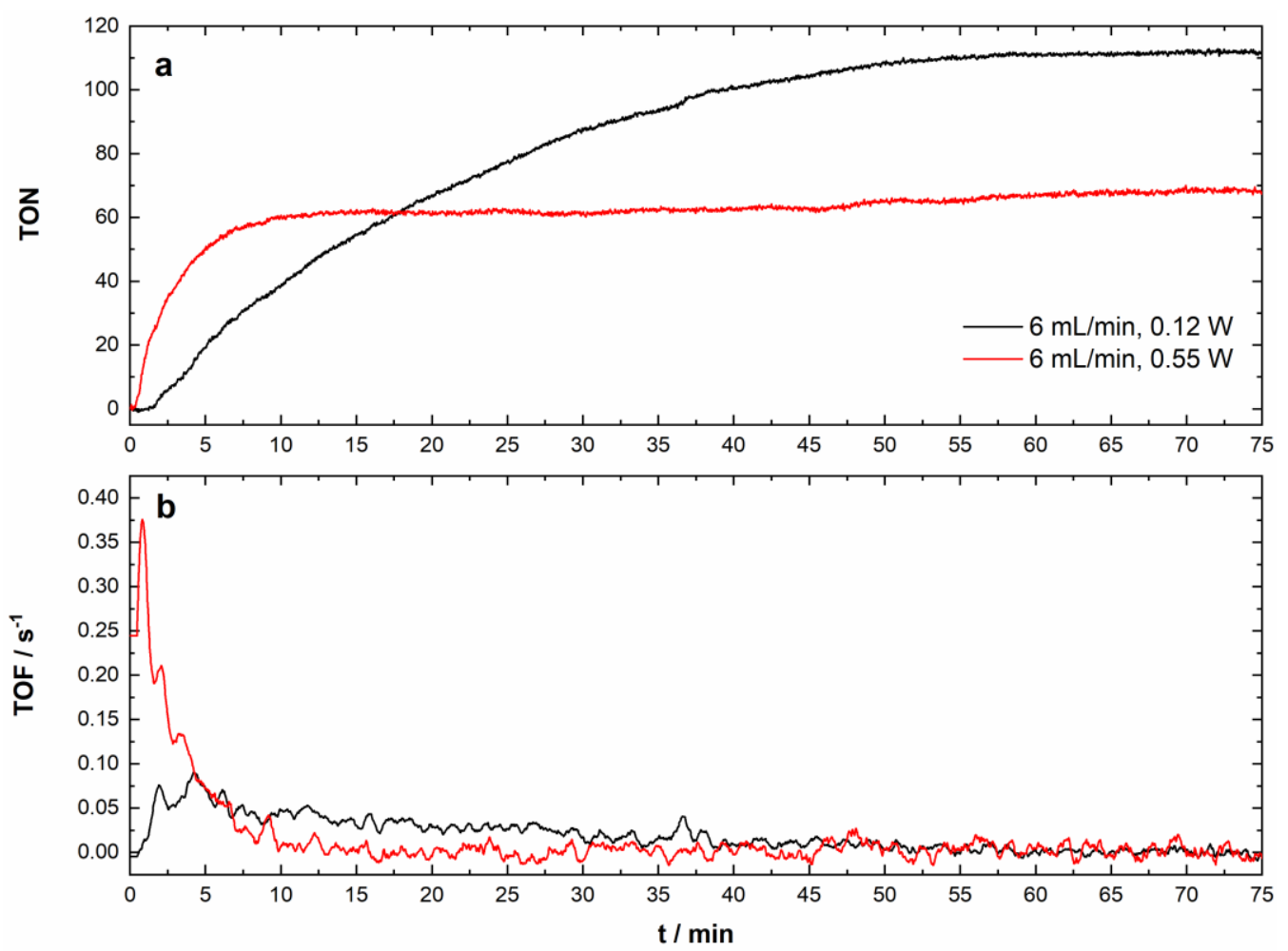

Figure 3: Comparison of TON (a) and TOF (b) between measurements at $0.12 \mathrm{~W}$ (black) and $0.55 \mathrm{~W}$ (red) radiant power, conducted at a flow rate of $6 \mathrm{~mL} \mathrm{~min} \mathrm{~m}^{-1}$.

To understand the factors determining the activity and long-term stability, further investigations were pursued. For higher flow rates, not only shorter irradiation and dark phases have to be considered, but also induction of secondary flows such as Dean vortices that accelerate radial convective mass transport in the capillaries. ${ }^{[26]}$ For the used reaction conditions, steep intensity gradients exist in the irradiation zone, i.e., the intensity drops by about $70 \%$ within the first $10 \%$ of the capillary cross section. Hence, regions exist that are not irradiated, while other regions absorb a large photon flux. Enhanced radial mass transport may compensate for regions that absorb a large photon flux through intimate material exchange between irradiated regions close to the light source and dark regions. Optimal conditions were found for flow rates of $29 \mathrm{~mL} \mathrm{~min}^{-1}$ and above for the investigated irradiation intensity range.

It is very clear from these analyses, that excess photons are detrimental for the overall performance, driving competing side reactions such as ligand photosubstitution of the ruthenium polypyridine PS. [27-30] Beside this, oxidative degradation of the PS represents another degradation pathway, as outlined by Bonnet et al. for related [Ru(bpy $)_{3}{ }^{2+}$ ] serving as model photosensitizer, PSm (see Figure 4). ${ }^{[31]}$ PSm $^{+}$ was found to be further oxidated by an hydroxide anion, generated in almost neutral ( $\mathrm{pH}$ 6.5) environment, through an oxidative attack on one of the $\alpha$-carbon atoms at the bipyridine sphere (PSm$\mathrm{OH}$ ). This starts an oxidative degradation cascade of the PSm, fueled by competing $\mathrm{PSm}^{+}$and the sacrificial electron acceptor. High photon fluxes and high amounts of $\mathrm{S}_{2} \mathrm{O}_{8}{ }^{2-}$ as sacrificial electron acceptor were reasoned by the authors to cause an accumulation of $\mathrm{PSm}^{+}$that drives this cascade and thus leads to fast deactivation of the catalysis. ${ }^{[31]}$ 


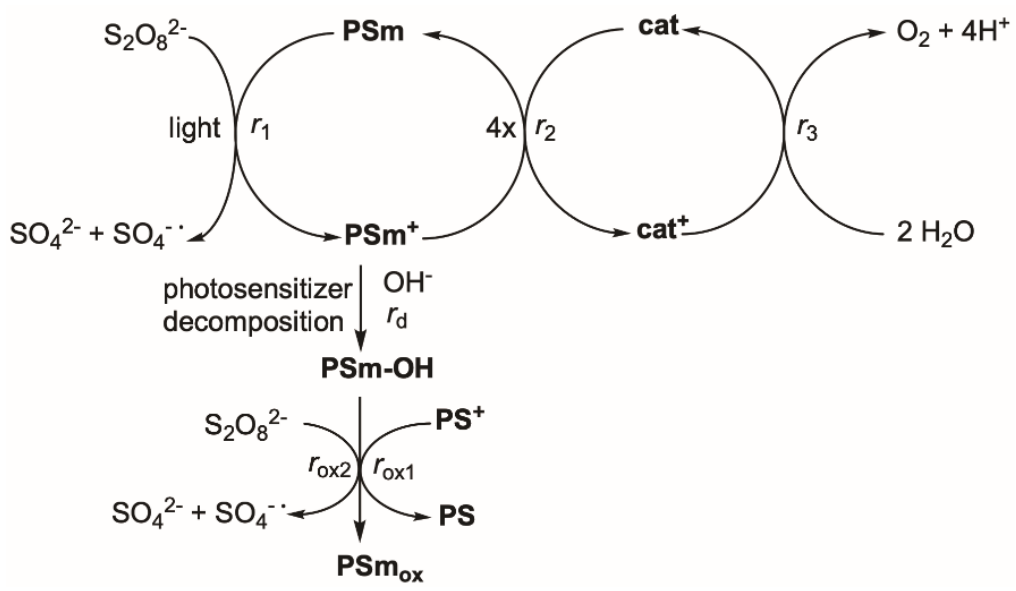

Figure 4: Simplified scheme of light-driven water oxidation. The rate of the reactions 1,2 and 3 is determined by the rates $r_{1}$ formation rate of $\mathbf{P S m}^{+}, r_{2}$ electron transfer rate from cat to $\mathbf{P S m}^{+}$and $r_{3}$ rate of the oxygen production at the water oxidation catalyst. Proposed decomposition pathway of PSm determined by the slowest reaction. The PSm decomposition is characterized by the rate $r_{d}$. The decomposition Product of PSm, PSm-OH is oxidized with the rates $r_{0 \times 1}$ and $r_{\text {oxz }}$ by reacting with $\mathbf{P S m}^{+}$or $\mathrm{S}_{2} \mathrm{O}_{8}{ }^{2-}$. Figure adapted from Bonnet et al. [32]

To investigate the fate of the PS, $\left[\mathrm{Ru}(\mathrm{dceb})_{2}(\mathrm{bpy})\right]\left(\mathrm{PF}_{6}\right)_{2}$ Figure $1 \mathrm{a}$, employed here, with a different substitution pattern compared to the model PSm, we investigated the change of its optical spectroscopic properties under catalytic conditions. PS shows visible emission with a maximum at $635 \mathrm{~nm}$ (Fig. S7), while Ru(dpp) is not emissive. ${ }^{[15]}$ As an indicator for functionality of the PS, emission of the reaction solution was measured with a clamp-on emission cell (see ESI) in situ. A comparison of TOF and emission intensity is shown in Figure 3. Within 90 minutes, the emission drops to about $10 \%$ of its original intensity and clearly correlates with the decreasing TOF. To further support this observation, ex situ UV-vis spectroscopic investigation under catalytic conditions were conducted in inert cuvettes at different irradiation intensities $(0.12 \mathrm{~W}$ and $0.55 \mathrm{~W})$. The results show faster degradation of the PS at higher irradiation intensities depicted in Figure 6 and Figure 7. In good correlation to the emission measurements, the MLCT band at $482 \mathrm{~nm}$ drops to approximately $13 \%$ of its former intensity.

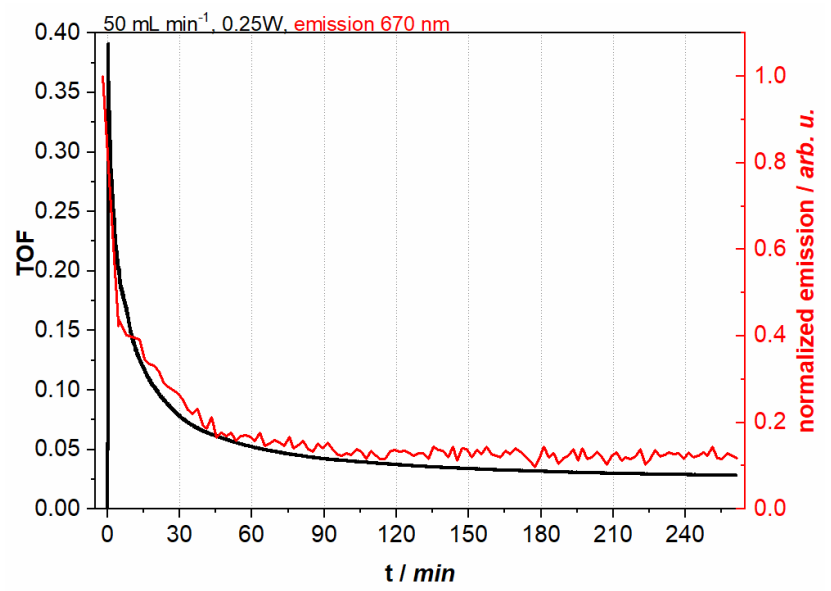

Figure 5. Luminescence at $670 \mathrm{~nm}$ (red, see Fig. S7 for emission spectrum of the PS) and TOF over time (black). Measurements were performed at $50 \mathrm{~mL} \mathrm{~min}{ }^{-1}$ and $0.25 \mathrm{~W}$. 

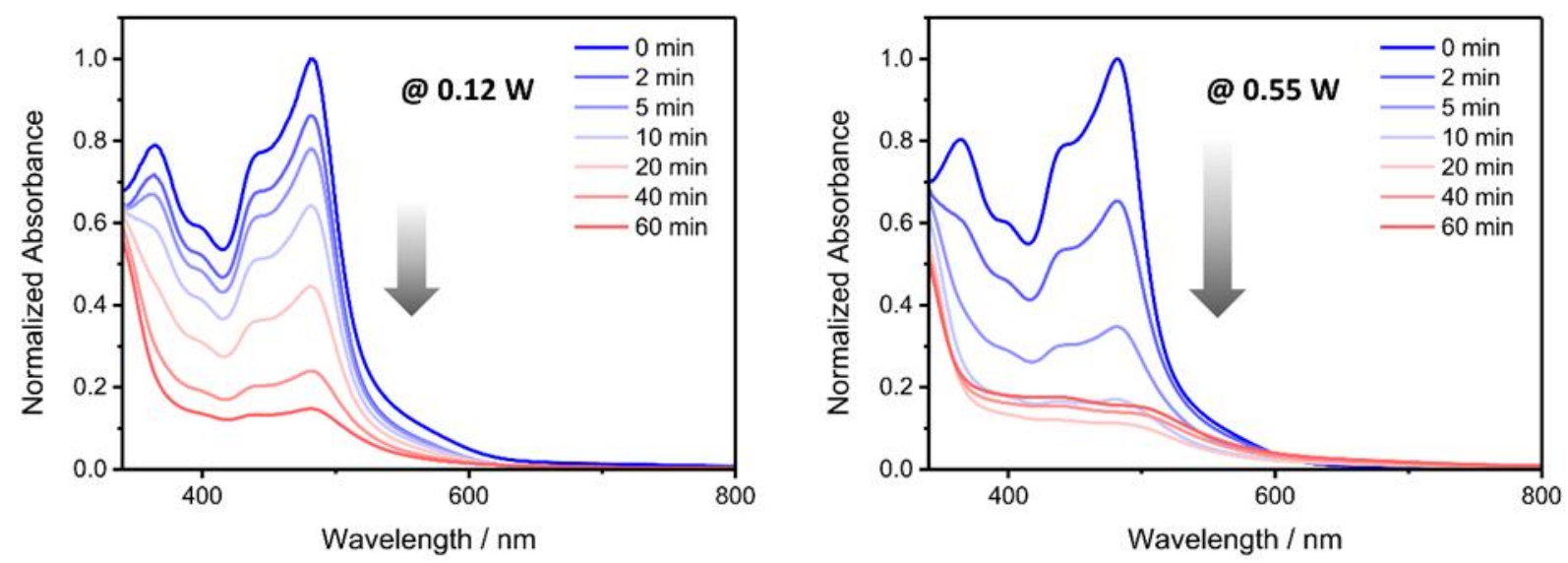

Figure 6: Decrease of absorbance of PS over the course of 1 hour at $0.12 \mathrm{~W}(0.043 \mathrm{~A})$ radiant power (left) compared to 0.55 $W(0.344 \mathrm{~A})$ (right) for the same time frame.

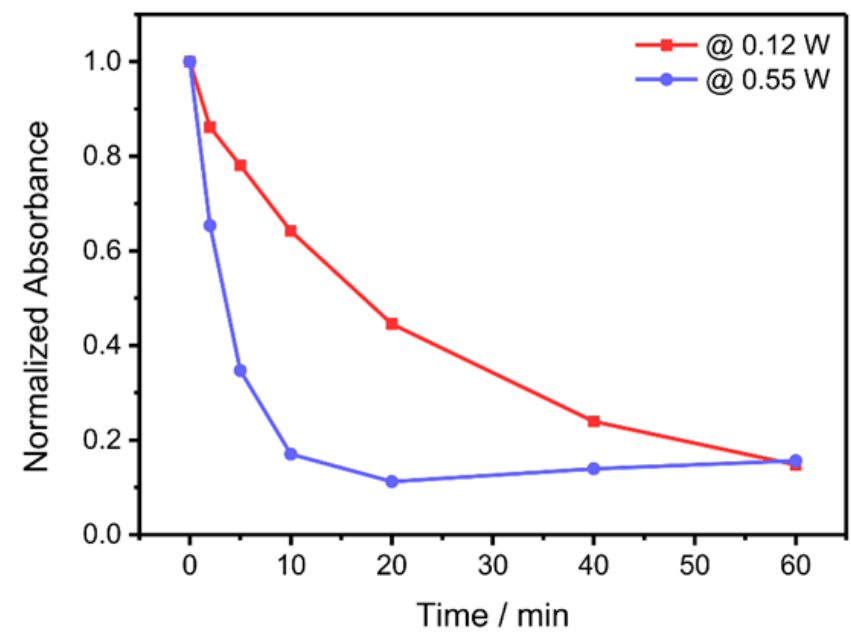

Figure 7: Time-resolved decrease of MLCT absorbance maximum at $482 \mathrm{~nm}$ of catalysis solutions at $0.12 \mathrm{~W}(0.043 \mathrm{~A})$ and 0.55 $W(0.344 \mathrm{~A})$.

To understand the molecular origin of the observed deactivation, we employed Raman spectroscopy as a label-free spectroscopic tool with high molecular specificity. The Raman spectra of the pure buffer, and difference spectra of the sacrificial electron acceptor $\mathrm{Na}_{2} \mathrm{~S}_{2} \mathrm{O}_{8}$ and the catalytic mixture are depicted in Figure 8. Both sacrificial electron acceptor and PS can be clearly identified on the buffer background. Due to its low concentration, the catalyst Ru(dpp) cannot be monitored via Raman spectroscopy (see ESI for spectra) and does not exhibit any visible Raman signal. The signal at $1075 \mathrm{~cm}^{-}$ ${ }^{1}$ is indicative specifically for the $\mathrm{S}_{2} \mathrm{O}_{8}{ }^{2-}$ ion, for PS a range of signals in the fingerprint region (1200 $1700 \mathrm{~cm}^{-1}$ ) are assignable to various vibrations of the bpy ligands and weaker signals between 300 and $350 \mathrm{~cm}^{-1}$ can be assigned to vibrations involving the Ru-N bonds (assignment is supported by densityfunctional theory calculations, see ESI).

These regions of interest were subsequently analyzed in situ in a cuvette containing the catalytic mixture by irradiation with a $455 \mathrm{~nm}$ LED ( $P_{\text {Light }}=95 \mathrm{~mW}$ or $700 \mathrm{~mW}$, see Figure 9). Upon irradiation, a 
rapid loss of PS signals in the fingerprint as well as the Ru-N region can be observed for both experiments, indicating the loss of bipyridine ligands from the ruthenium center. This process is even more rapid for the high-power experiment, indicating a photon flux dependent reaction. Furthermore, especially for the high-power experiment (Figure 9, left) a clear loss of the persulfate band can be observed which coincides with the appearance of a new band at $980 \mathrm{~cm}^{-1}$ that can be assigned to a sulfate vibration, i.e., indicating reduction of the sacrificial electron acceptor and therefore catalytic activity.

Kinetic profiles of the Raman measurements allow to make two crucial observations about the system (see Figure 10, see ESI for calculations). First, the loss of Ru-N signal and "bpy"-signal happens concertedly. This corroborates a degradation mechanism that involves loss of ligands as deactivation path, rather than a mechanism involving chemical changes of the "bpy"-ligands while it stays attached to the ruthenium center for prolonged periods of time. Second, the apparent PS degradation and catalytic activity are highly decoupled: While the signal intensity for the PS is lost almost completely in the first minute (high power) or 5 minutes (low power), the accumulation of sulfate continues for much longer periods of time until flattening out after around 15 minutes (high power) and 70 minutes (low power), respectively.

A likely explanation for this behavior is a transport limited reaction around the photosensitizer: The reaction of PS with $\mathrm{S}_{2} \mathrm{O}_{8}{ }^{2-}$ produces a sulfate ion as well as a sulfate radical, which is an extremely potent oxidant capable of oxidizing even aromatic structures. ${ }^{[33-35]}$ This sulfate radical is the likely cause for the oxidative ligand substitution proposed as a degradation mechanism (see Figure 4). ${ }^{[31]}$ For high concentrations of PS, it is more likely that diffusion of $\mathrm{SO}_{4}{ }^{\bullet-}$ leads to collision with another PS molecule, thereby initiating oxidative decomposition. After the initial drop in PS concentration observed in the Raman spectra (and in UV-vis as well as emission spectroscopic investigations), the probability of the collision between the highly reactive $\mathrm{SO}_{4}{ }^{\circ-}$ and PS drops significantly. Therefore, stabilizing the relatively low concentration of PS enables sustained catalytic activity. This data furthermore implies that the initial light driven electron transfer between the PS and $\mathrm{S}_{2} \mathrm{O}_{8}{ }^{2-}$ does not lead to immediate attack of the formed $\mathrm{SO}_{4}{ }^{\circ-}$ on the oxidized PS in its spatial vicinity, since the low concentration of PS is stable for extended periods of catalysis with continuous formation of $\mathrm{SO}_{4}{ }^{2-}$. Recently performed detailed investigations into the light driven and thermal oxidation of ruthenium complexes with $\mathrm{S}_{2} \mathrm{O}_{8}{ }^{2-}$ suggested a fast dissociation of the formed $\mathrm{SO}_{4}{ }^{-{ }^{-}}$from the metal cation, ${ }^{[36]}$ leading to a decoupling of both $\mathrm{PS}$ degradation and $\mathrm{SO}_{4}{ }^{2-}$ formation kinetics. This proposed mechanism is supported by the kinetic plots for the low power experiment (see Figure 8). Here, a flattening of the $\mathrm{SO}_{4}{ }_{4}{ }^{2-} / \mathrm{S}_{2} \mathrm{O}_{8}{ }^{2-}$ can be seen at around 70 minutes, which coincides with the PS band areas reaching values very close to 0 . Until this point, PS is still present inside the catalytic mixture, only degrading relatively slowly and only stopping the catalytic activity after complete degradation.

Furthermore, the degradation of PS is less dependent on the radiant power compared to the $\mathrm{SO}_{4}{ }^{2-}$ $/ \mathrm{S}_{2} \mathrm{O}_{8}{ }^{2-}$ kinetic, indicating that PS degradation is transport limited. An endpoint analysis after 60 minutes (high power, see Fig S11) and 800 minutes (low power, see Figure 8) together with DFT calculations (details see ESI) revealed the following insights: First, a complete loss of Ru-N intensity can be observed, without appearance of new low-frequency signals in this region, indicating that the degradation pathway ends in a ruthenium species with a significantly lower Raman activity e.g., $\mathrm{Ru}\left(\mathrm{H}_{2} \mathrm{O}\right)$ complexes. Second, the fingerprint region shows a loss of band structure but retains the general position of the most intense bands with only slight changes to position and band shape. This makes it likely that the degraded mixture still contains bpy like structures. The loss of some bands can be explained by a significant decrease of Raman activity upon detachment from the Ru-center, which makes weaker bands difficult to detect, and the loss of geometric arrangement around the ruthenium, which removes bands induced by the geometric distortion due to metal-ligand bonds. 


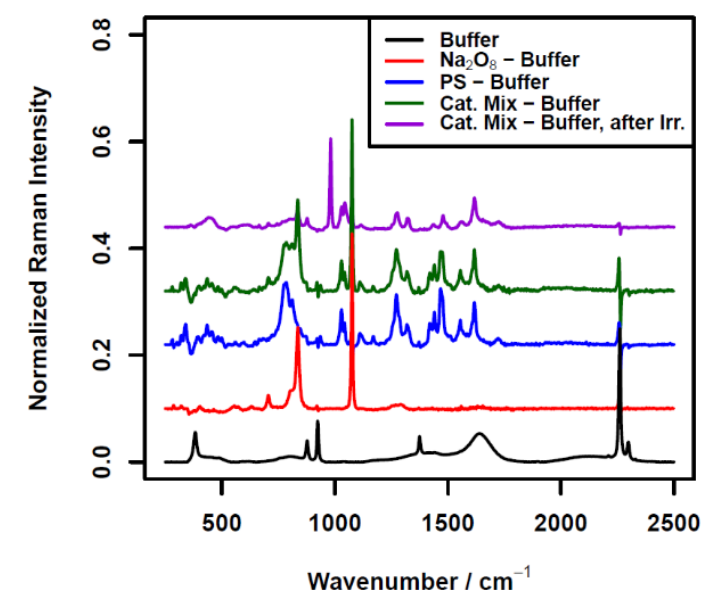

Figure 8: Normalized (to MeCN vibration @ $2260 \mathrm{~cm}^{-1}$ ) Raman spectra $\left(\lambda_{\mathrm{Exc}}=785 \mathrm{~nm}\right)$ of $\mathrm{H}_{3} \mathrm{BO}_{3} / \mathrm{NaHCO}_{3}$ buffer (black, $\mathrm{pH}=6.5,0.08 \mathrm{M} \mathrm{H}_{3} \mathrm{BO}_{3}, \mathrm{H}_{2} \mathrm{O} / \mathrm{MeCN}, 96: 4 \mathrm{v} / \mathrm{v}$ ) and difference Raman spectra against the buffer of solutions with added $\mathrm{Na}_{2} \mathrm{~S}_{2} \mathrm{O}_{8}$ (red, $10 \mathrm{mM}$ ) and PS (blue, $0.3 \mathrm{mM}$ ), the catalytic mixture (10 mM Na${ }_{2} \mathrm{~S}_{2} \mathrm{O}_{8}, 0.3 \mathrm{mM}$ PS, $2.6 \mu \mathrm{M}$ Ru(dpp)) before (green) and after irradiation (purple, $455 \mathrm{~nm} L E D, 95 \mathrm{~mW}, 800 \mathrm{~min}$ ).
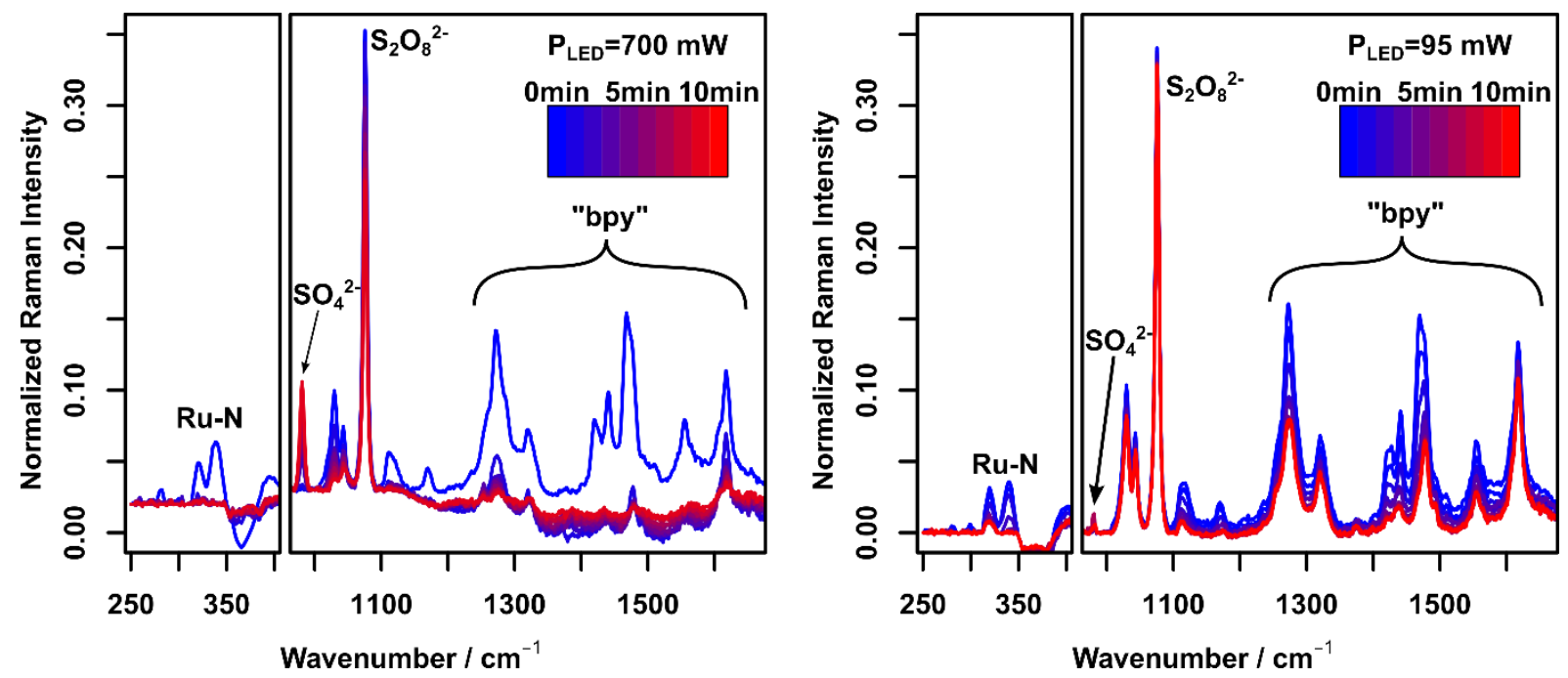

Figure 9: Normalized (to MeCN vibration @ $2260 \mathrm{~cm}^{-1}$, not shown) difference Raman spectra ( $\lambda_{E x c}=785 \mathrm{~nm}$, difference against buffer, see Figure X1) of a catalytic mixture containing $10 \mathrm{mM} \mathrm{Na} \mathrm{S}_{2} \mathrm{O}_{8}, 0.3 \mathrm{mM}$ PS and $2.6 \mu \mathrm{M} \mathrm{Ru}(\mathrm{dpp})$ in the time range of 10 minutes after starting irradiation with a $455 \mathrm{~nm}$ LED and radiant powers of $700 \mathrm{~mW}$ (left) and $95 \mathrm{~mW}$ (right), respectively. 
$P_{\text {LED }}=700 \mathrm{~mW}$

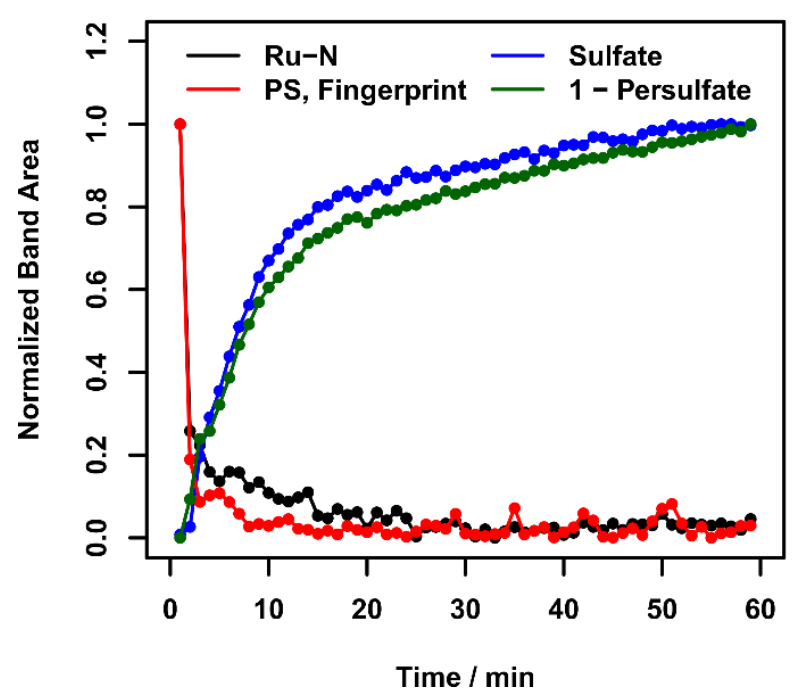

$P_{\text {LED }}=95 \mathrm{~mW}$

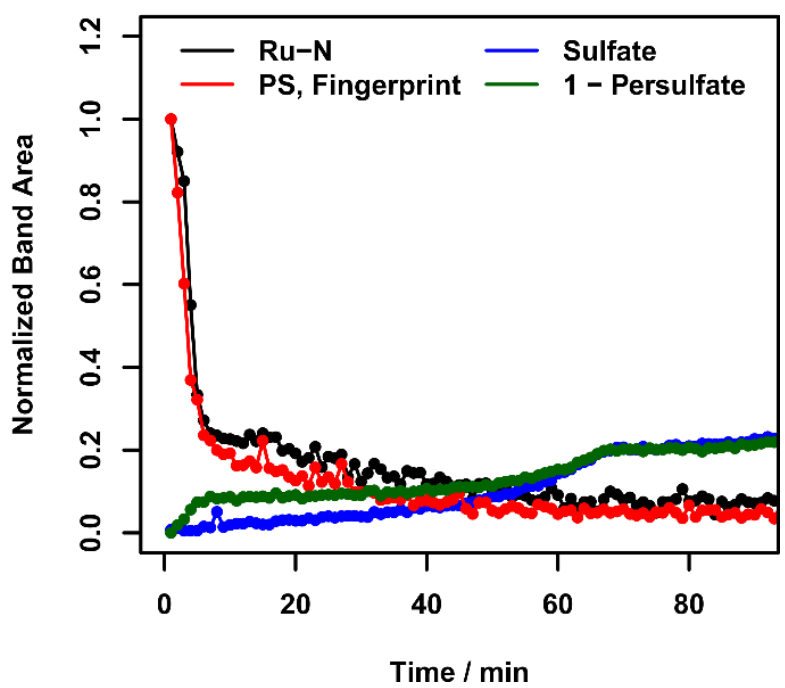

Figure 10: Kinetic profiles calculated from integrated band areas of selected regions of interest of a catalytic mixture containing $10 \mathrm{mM} \mathrm{Na} \mathrm{S}_{2} \mathrm{O}_{8}, 0.3 \mathrm{mM}$ PS and $2.6 \mu \mathrm{M} \mathrm{Ru(dpp)}$ (details see text) in the time range of 60/80 minutes after starting irradiation with a $455 \mathrm{~nm}$ LED and radiant powers of $700 \mathrm{~mW}$ (left) and $95 \mathrm{~mW}$ (right), respectively.

To gain a deeper understanding on the crucial kinetics of the $\mathrm{S}_{2} \mathrm{O}_{8}{ }^{2-}$ consumption and $\mathrm{SO}_{4}{ }^{2-}$ formation, online IR spectroscopy using the developed flow reactor was employed to avoid interference between the signatures of the PS and WOC, and to ensure the same irradiation/reaction conditions as for the catalytic studies (detailed description see ESI). For different irradiation intensities (i.e., $0.12 \mathrm{~W}$ and $0.55 \mathrm{~W}$ ), the IR studies on the photocatalytic reactions yielded results that independently confirm the Raman investigations, i.e., a decrease of the $\mathrm{S}_{2} \mathrm{O}_{8}{ }^{2-}$ signal intensity (at $1287 \mathrm{~cm}^{-1}$ ), and correspondingly, a gain in $\mathrm{SO}_{4}{ }^{2-}$ signal intensity (at $1116 \mathrm{~cm}^{-1}$ ) (Figure 11). ${ }^{[37,38]}$
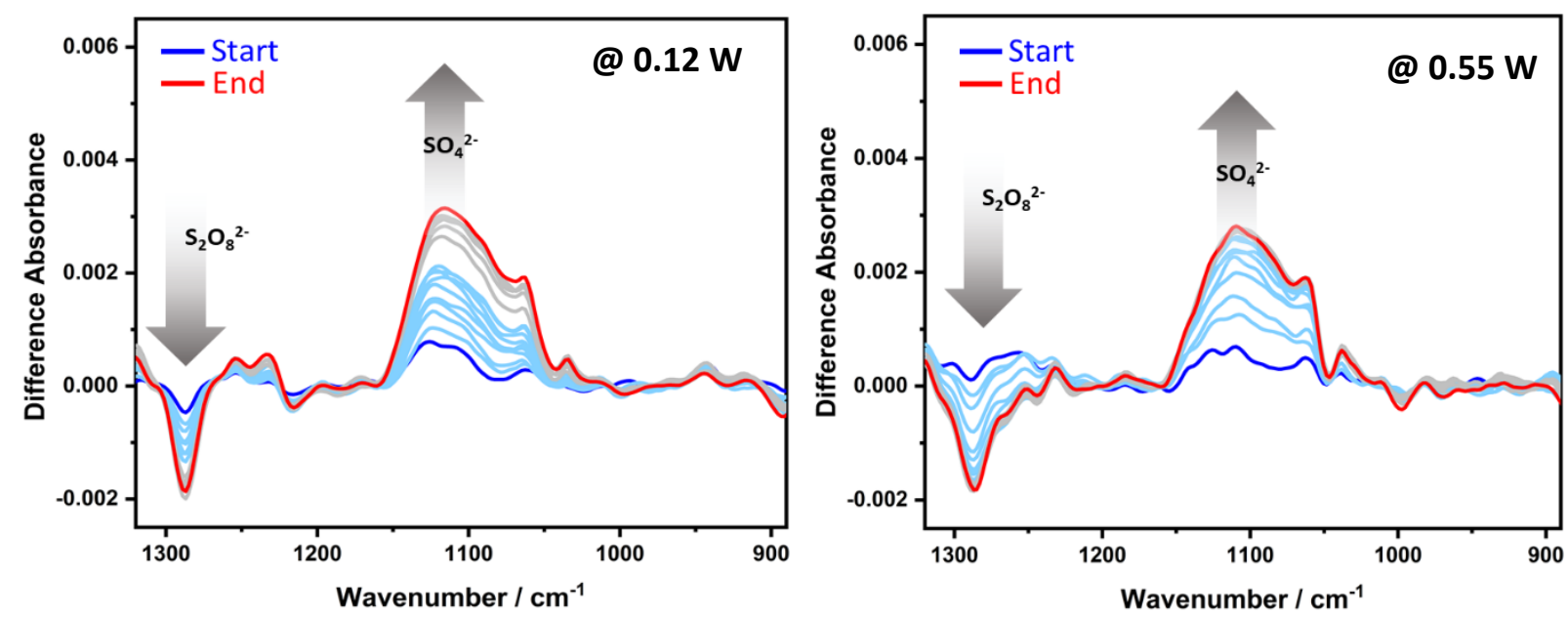

Figure 11: Difference IR-ATR absorbance spectra for radiant power of $0.12 \mathrm{~W}$ (left) and $0.55 \mathrm{~W}$ (right). Molecular changes within the first 15 min are shown in light blue. Dark blue indicates the first spectrum, red indicates the final spectrum (i.e., after $60 \mathrm{~min})$.

However, upon monitoring the kinetics of the changes of the signal intensity for both species in dependence on the irradiation intensity, a difference is evident compared to the Raman results for the kinetics of the decrease in $\mathrm{S}_{2} \mathrm{O}_{8}{ }^{2-}$ signal vs. the increase in $\mathrm{SO}_{4}{ }^{2-}$ signal (see Figure 12). The increase of the $\mathrm{SO}_{4}{ }^{2-}$ signal is faster at irradiation with higher intensity (i.e., at $0.55 \mathrm{~W}$ ) compared to lower intensity (i.e., at $0.12 \mathrm{~W}$ ). This is evident from the different slopes during the first $15 \mathrm{~min}$ in Figure $12 \mathrm{~b}$ ). Afterwards, the changes are less evident at all irradiation intensities. In fact, the $\mathrm{S}_{2} \mathrm{O}_{8}{ }^{2-}$ is anticipated to decrease with time, as it acts as sacrificial electron acceptor, and is therefore consumed during the 
catalytic reaction and converted into $\mathrm{SO}_{4}{ }^{2-}$. While this is clearly evident in the IR spectra (see Figure $12 \mathrm{a})$ ), the kinetic of the $\mathrm{SO}_{4}{ }^{2-}$ formation is different. For the higher and the lower irradiation intensity, the $\mathrm{S}_{2} \mathrm{O}_{8}{ }^{2-}$ signal is decreasing equally fast. $\mathrm{As}_{2} \mathrm{O}_{8}{ }^{2-}$ reveals less intense IR bands and the temporal evolution of the $\mathrm{SO}_{4}{ }^{2-}$-signal is in line with the time resolved oxygen measurements (see Figure 3 ), it may be concluded that the differences in catalytic activity at various irradiation intensities are more facilely followed via the sulfate band during IR spectroscopic studies.
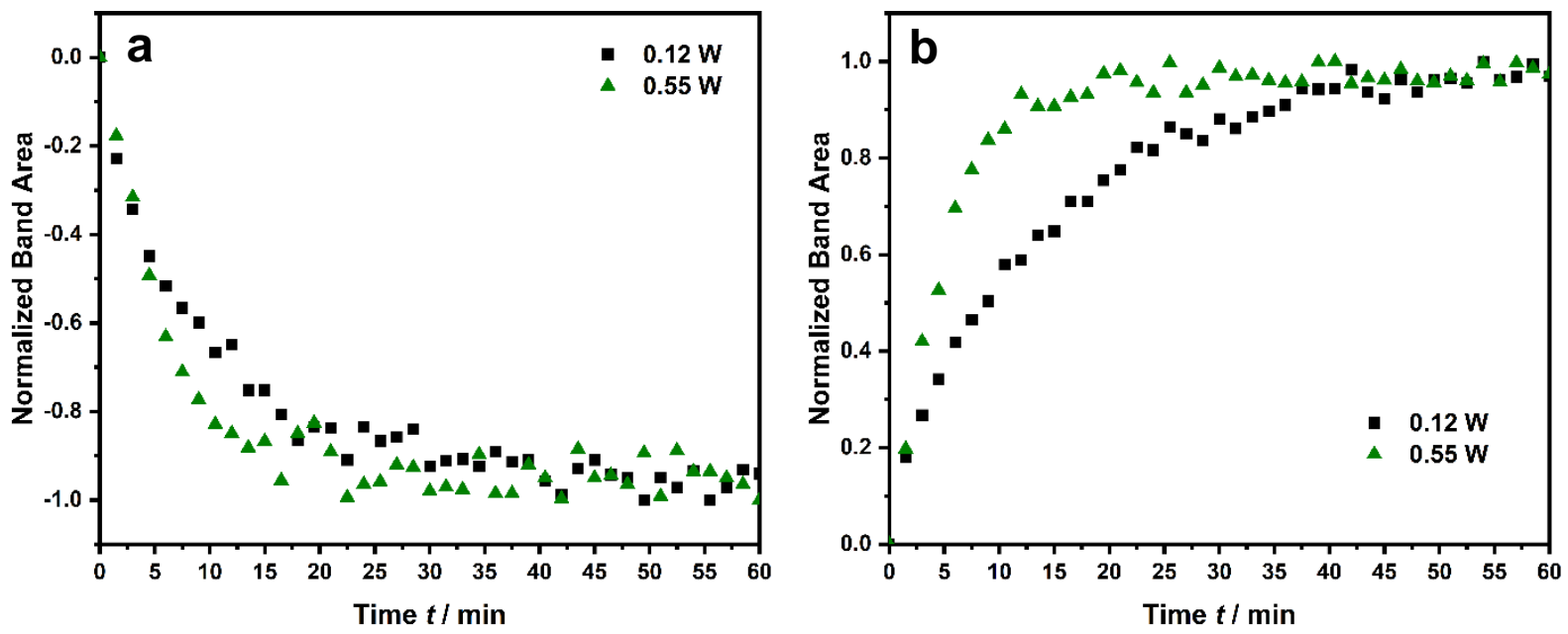

Figure 12: Integrated peak area of (a) the $\mathrm{S}_{2} \mathrm{O}_{8}{ }^{2-}$ difference band with time, and (b) the $\mathrm{SO}_{4}{ }^{2-}$ difference band at different irradiation intensities.

The kinetic studies give evidence that degradation of the PS is limiting the performance of the lightdriven WOC system under investigation. Accumulation of $\mathrm{PS}^{+}$together with the presence of $\mathrm{SO}_{4}{ }^{\circ}$ open the degradation pathways. Lower irradiation intensities reduce the rate of PS excitation and thus the accumulation of PS $^{+}$finally leading to slower PS degradation.

Lower irradiation intensities can also be realized, when the temporal availability of photons is controlled, leading to an on average lower photon flux. In the used flow setup, higher flow rates lead to shorter irradiation and dark periods, while the ratio between both periods stays the same. In addition, higher flow rates lead to enhanced mass transport along the irradiation direction. ${ }^{[39]}$ Correlating the TON against the radiant power (see Figure 2 a) shows similar trends for all flow rates. Lower photon fluxes are leading to the already discussed higher TONs. Interestingly, higher flow rates amplify this effect even further. This is attributed to the increased radial fluid movement perpendicular to the flow direction in the capillaries that occur at higher flow rates. ${ }^{[26,40]}$ Note that the flow conditions are always laminar (Reynolds number between 80 and 670 ) and temporal fluctuations of the flow field that are characteristic for turbulent flows do not occur. The degree of absorption is about $80 \%$ in the used capillary (absorbance of about 0.7). Hence, a strong intensity gradient exists along the light ray trajectory and thus high intensities close to the irradiated wall and very low intensities close to the opposite wall. Radial fluid movement, accelerated by Dean vortices (Dean numbers of 401 to 3341 were calculated for the system, see ESI for further details), will transport small volume units away from the light source and with this leads to an on average reduced availability of photons.

To further investigate the influence of mass transport, a reference experiment was conducted by irradiating the catalytic solution in a closed vial under stirred and not-stirred conditions. ${ }^{[18]}$ The larger dimension of the closed vial setup leads to an even more pronounced formation of dark zones. This situation may be utilized to homogenize the availability of photons to the PS along the light ray trajectory by intense mixing. The rotational movement of the liquid together with the steep intensity gradients will result in a dynamically changing irradiation. A reference volume is first transported to the irradiation zone close to the LED. The more this volume approximates the LED, the higher the 
photon flux is, which is absorbed in this volume. Subsequently, the volume is transported into the dark region, associated with a decrease of the incident photon flux. To elucidate this effect, a custom-made stirrer was used to ensure intense stirring of the entire solution. The apparent TON is roughly doubled from around 110 to 230 when stirring the solution (see ESI). These observations strongly support the hypothesis that mass transport enhances the overall performance of light driven reactions by changing the temporal availability of photons.

\section{Conclusions}

In our study we show that photon flux management is a powerful tool to bring photocatalytic water splitting reaction performance to a new level. By reducing the temporal availability of photons through appropriate reactor design or enhanced mass transport along the light ray trajectory, an improved synchronization of different reaction steps is achieved. Furthermore, detailed in operando investigations of the photocatalytic mixtures with complementary spectroscopic methods suggest an additional photocatalytic activity limiting reaction pathway. Loss of PS as validated by UV-vis, Raman and emission spectroscopy is caused by attack of $\mathrm{SO}_{4}{ }^{-}$on PS in close vicinity, i.e. during the initial phase of photocatalysis. Monitoring rise of $\mathrm{SO}_{4}{ }^{2-}$ concentration and decline of $\mathrm{S}_{2} \mathrm{O}_{8}{ }^{2-}$ utilizing IR and Raman spectroscopy does show that the significantly lower concentration of PS is active and stable over prolonged time of catalysis. Therefore, attack of the $\mathrm{SO}_{4}{ }^{-0}$, formed after the reduction of $\mathrm{S}_{2} \mathrm{O}_{8}{ }^{2-}$ by the excited PS, at the newly generated $\mathbf{P S}^{+}$in its immediate vicinity is not the dominant pathway. Minimizing the decomposition of PS through reducing the rate of excitation results in a 11 -fold increase in TON. This effect is even more pronounced for the external photonic efficiency with an increase by a factor of 31, effectively improving the efficiency of light harvesting by more than an order of magnitude.

Thus, we show for the first time the synergistic effect of reaction engineering in light driven water splitting reactions with molecular catalysts. With ever more elaborated (photo)catalysts being developed, it is clear that tuning reaction conditions on all relevant scales will play a crucial part in achieving maximum performance of the catalytic system, especially in view of the future industrial application of such processes. While the complex apparatus of biological photosynthesis features mechanisms to synchronize different reactions steps and protect or repair light-harvesting units, this is often not the case in artificial photosynthesis. ${ }^{[41-44]}$ Here the oxidative half reaction usually proceeds on a much shorter timescale than the reductive half reaction. ${ }^{[45]}$ Smart reaction design and engineering will help to overcome these limitations.

\section{Acknowledgements}

This research was funded by the Deutsche Forschungsgemeinschaft DFG as part of the collaborative research center TRR234 "CataLight" (364549901), project A1, A4, C2 and C6.

Keywords: photocatalysis $\bullet$ photoreaction engineering $\bullet$ water oxidation $\bullet$ flow-chemistry $\bullet$ ruthenium

\section{References}

[1] M. Oelgemöller, Chem. Rev. 2016, 116, 9664-9682.

[2] D. Cambie, C. Bottecchia, N. J. W. Straathof, V. Hessel, T. Noe, Chem. Rev. 2016, 116, 10276-10341.

[3] N. S. Lewis, D. G. Nocera, Proc. Natl. Acad. Sci. U. S. A. 2006, 103, 15729-15735.

[4] A. Kudo, Y. Miseki, Chem. Soc. Rev. 2009, 38, 253-278.

[5] R. Matheu, P. Garrido-Barros, M. Gil-Sepulcre, M. Z. Ertem, X. Sala, C. Gimbert-Suriñach, A. Llobet, Nat. Rev. Chem. 2019, 3, 331341.

[6] J. Ran, J. Zhang, J. Yu, M. Jaroniec, S. Z. Qiao, Chem. Soc. Rev. 2014, 43, 7787-7812.

[7] W. T. Eckenhoff, R. Eisenberg, Dalt. Trans. 2012, 41, 13004-13021.

[8] M. D. Kärkäs, O. Verho, E. V. Johnston, B. Åkermark, Chem. Rev. 2014, 114, 11863-12001.

[9] M. Schulz, M. Karnahl, M. Schwalbe, J. G. Vos, Coord. Chem. Rev. 2012, 256, 1682-1705. 
[10] D. Ziegenbalg, A. Pannwitz, S. Rau, B. Dietzek-Ivanšić, C. Streb, 2021, DOI 10.5281/zenodo.5575037.

[11] A. N. Tikhonov, Cell Biochem. Biophys. 2017, 75, 421-432.

[12] F. Guba, Ü. Tastan, K. Gugeler, M. Buntrock, T. Rommel, D. Ziegenbalg, Chemie-Ingenieur-Technik 2019, 91, 17-29.

[13] I. Reim, B. Wriedt, Ü. Tastan, D. Ziegenbalg, M. Karnahl, ChemistrySelect 2018, 3, 2905-2911.

[14] S. Meyer, D. Tietze, S. Rau, B. Schäfer, G. Kreisel, J. Photochem. Photobiol. A Chem. 2007, 186, $248-253$.

[15] R. Zong, R. P. Thummel, J. Am. Chem. Soc. 2004, 126, 10800-10801.

[16] L. Tong, R. Zong, R. Zhou, N. Kaveevivitchai, G. Zhang, R. P. Thummel, Faraday Discuss. 2015, 185, 87-104.

[17] J. T. Muckerman, M. Kowalczyk, Y. M. Badiei, D. E. Polyansky, J. J. Concepcion, R. Zong, R. P. Thummel, E. Fujita, Inorg. Chem. 2014, 53, 6904-6913.

[18] F. L. Huber, S. Amthor, B. Schwarz, B. Mizaikoff, C. Streb, S. Rau, Sustain. Energy Fuels 2018, 2, $1974-1978$.

[19] J. P. Knowles, L. D. Elliott, K. I. Booker-Milburn, Beilstein J. Org. Chem. 2012, 8, 2025-2052.

[20] C. Sambiagio, T. Noël, Trends Chem. 2020, 2, 92-106.

[21] K. Loubière, M. Oelgemöller, T. Aillet, O. Dechy-Cabaret, L. Prat, Chem. Eng. Process. - Process Intensif. 2016, 104, 120-132.

[22] L. D. Elliott, J. P. Knowles, P. J. Koovits, K. G. Maskill, M. J. Ralph, G. Lejeune, L. J. Edwards, R. I. Robinson, I. R. Clemens, B. Cox, D. D. Pascoe, G. Koch, M. Eberle, M. B. Berry, K. I. Booker-Milburn, Chem. - A Eur. J. 2014, 20, DOI 10.1002/chem.201404347.

[23] M. Sender, D. Ziegenbalg, React. Chem. Eng. 2021, 6, 1614-1627.

[24] M. Sender, B. Wriedt, D. Ziegenbalg, React. Chem. Eng. 2021, 6, 1601-1613.

[25] M. G. Pfeffer, T. Kowacs, M. Wächtler, J. Guthmuller, B. Dietzek, J. G. Vos, S. Rau, Angew. Chemie - Int. Ed. $2015,54,6627-6631$.

[26] P. Hermann, J. Timmermann, M. Hoffmann, M. Schlüter, C. Hofmann, P. Löb, D. Ziegenbalg, Chem. Eng. J. 2018, 334, $1996-2003$.

[27] H. E. Bonfield, T. Knauber, F. Lévesque, E. G. Moschetta, F. Susanne, L. J. Edwards, Nat. Commun. $2020,11,804$.

[28] A. Soupart, F. Alary, J.-L. Heully, P. I. P. Elliott, I. M. Dixon, Inorg. Chem. 2020, DOI 10.1021/acs.inorgchem.0c01843.

[29] A. Vaidyalingam, P. K. Dutta, Anal. Chem. 2000, 72, 5219-5224.

[30] A. Juris, V. Balzani, F. Barigelletti, S. Campagna, P. Belser, A. von Zelewsky, Coord. Chem. Rev. 1988, 84, 85-277.

[31] B. Limburg, E. Bouwman, S. Bonnet, ACS Catal. 2016, 6, 5273-5284.

[32] B. Limburg, E. Bouwman, S. Bonnet, ACS Catal. 2016, 6, 5273-5284.

[33] P. Neta, V. Madhavan, H. Zemel, R. W. Fessenden, J. Am. Chem. Soc. 1977, 99, 163-164.

[34] J. Van Buren, A. A. Cuthbertson, D. Ocasio, D. L. Sedlak, Environ. Sci. Technol. Lett. 2021, 8, 574-580.

[35] S.-C. Hsu, T.-M. Don, W.-Y. Chiu, Polym. Degrad. Stab. 2002, 75, 73-83.

[36] A. Lewandowska-Andralojc, D. E. Polyansky, J. Phys. Chem. A 2013, 117, 10311-10319.

[37] D. J. Cziczo, J. P. D. Abbatt, J. Geophys. Res. Atmos. 1999, 104, 13781-13790.

[38] H. A. Elwan, M. T. Zaky, A. S. Farag, F. S. Soliman, M. Ezel Dean Hassan, J. Mol. Liq. 2017, 248, 549-555.

[39] F. Guba, F. Gaulhofer, D. Ziegenbalg, J. Flow Chem. 2021, DOI 10.1007/s41981-021-00174-1.

[40] F. Schönfeld, S. Hardt, AlChE J. 2004, 50, 771-778.

[41] L. T. Hickey, A. N. Hafeez, H. Robinson, S. A. Jackson, S. C. M. Leal-Bertioli, M. Tester, C. Gao, I. D. Godwin, B. J. Hayes, B. B. H. Wulff, Nat. Biotechnol. 2019, 37, 744-754.

[42] N. C. Rockwell, Y. S. Su, J. C. Lagarias, Annu. Rev. Plant Biol. 2006, 57, 837-858.

[43] A. I. Velez-Ramirez, W. Van leperen, D. Vreugdenhil, P. M. J. A. Van Poppel, E. Heuvelink, F. F. Millenaar, Nat. Commun. 2014, 5, DOI 10.1038/ncomms5549.

[44] A. I. Velez-Ramirez, W. Van leperen, D. Vreugdenhil, F. F. Millenaar, Trends Plant Sci. 2011, 16, 310-318.

[45] R. Matheu, M. Z. Ertem, C. Gimbert-Suriñach, X. Sala, A. Llobet, Chem. Rev. 2019, 119, 3453-3471. 